\title{
APOYOS ECONÓMICOS-FISCALES EN MÉXICO POR COVID-19 PARA MIPYMES
}

\section{ECONOMIC-TAX SUPPORT IN MEXICO DUE TO COVID-19 FOR SMES}

\author{
Lic. Adriana Salas Ceballos a \\ Dra. Flor Lucila Delfín Pozos ${ }^{\text {b }}$ \\ Dra. María Pilar Acosta Márquez ${ }^{\text {c }}$ \\ Dr. Héctor Rogelio Olivares Galván d
}

\begin{abstract}
${ }^{a}$ Universidad Veracruzana Instituto de Investigaciones en Contaduría, adrianasalasceballos@gmail.com

${ }^{\mathrm{b}}$ Universidad Veracruzana Instituto de Investigaciones en Contaduría, fdelfin@uv.mx

${ }^{\mathrm{c}}$ Universidad Veracruzana Instituto de Investigaciones en Contaduría, pacosta@uv.mx

${ }^{\mathrm{d}}$ Universidad Veracruzana Instituto de Investigaciones en Contaduría, holivares@uv.mx
\end{abstract}

\section{RESUMEN}

A causa de la grave situación que está aconteciendo en el mundo por la pandemia Covid19, en el ámbito de salud, se puede destacar el económico, como uno de los más afectados, por el cese temporal de trabajos e incluso el cierre de empresas; las cuales no tienen los recursos suficientes para subsistir y la imposibilidad para solventar los gastos.

Ante este panorama se han realizado acciones por parte de las autoridades federales para frenar los despidos y el cierre de empresas. Algunos gobiernos estatales han otorgado apoyos a los empresarios MiPyMes para contrarrestar las consecuencias de esta situación.

El presente trabajo tiene como objetivo describir las medidas y posturas adoptadas por las autoridades en México durante el año 2020; presentando las decisiones económicas y fiscales con las que están intentando reducir las consecuencias ocasionadas por este hecho fortuito, enfocándose en las MiPyMes. Presentando las medidas adoptadas por los niveles de gobierno (estatal y federal) para distribuir los recursos que permita mitigar el desempleo y la crisis económica actual. 
La metodología que se empleará es cualitativa ya que se busca describir la situación del país, de acuerdo con las medidas adoptadas por las autoridades.

Con dicho análisis se puede concluir que las posturas adoptadas por las autoridades resultan insuficientes para contrarrestar la problemática generada por la pandemia mundial.

PAlabRAS ClAVE: Apoyos económicos; estímulos fiscales; MiPyMes; Pandemia covid-19.

\section{ABSTRACT}

Due to the precarious situation taking place in the world because of the COVID pandemic, the job market has been the one that has been the most affected. Massive firings and companies going out of business are the examples. The companies do not have enough resources to subsist and solve and spare expenses.

In order to face this scenery, the federal authorities have taken action in order to stop massive firings and business' closing. Some state governments have given financial support to small and large business in order to counteract the consequences.

The following writing intends to describe the measures and actions taken by the Mexican Authorities during the year 2020, presenting the legal and economic decisions made in order to control the precarious situations caused by this event, focusing in small and large businesses. We present here some measures taken by the levels of government (State and Federal) in order to distribute the resources that allow to lower unemployment and current economic crisis.

The researching methodology used will be qualitative, meaning it intends to describe the situation of the country, according to the actions taken by the authorities.

With such analysis, it can be concluded that the actions taken by the authorities turned out to be ineffective to control the problem caused by the world pandemic.

KEYWORDS: Economic support; fiscal incentives; SMEs; covid-19 pandemic. 


\section{INTRODUCCIÓN}

A causa de la pandemia generada por el coronavirus SARS-COV2 también llamado Covid19; la cual tuvo su aparición en China en diciembre de 2019, provocando que se extendiera por el mundo declarándose pandemia global por la Organización Mundial de la Salud en el 2020 (OMS, 2020a), y con el fin de mitigar los efectos ocasionados, las autoridades implementaron diferentes programas sociales de apoyo a la sociedad, entre ellos para las microempresas, los cuales son objeto de estudio en este trabajo. De modo que se inicia con la descripción de la situación actual en la que se encuentran las MiPyMes, las cuales requieren de la intervención de las autoridades para poder subsistir, sin tener la necesidad de despedir a sus empleados o cierre de las mismas, provocando estragos económicos mayores.

Con datos proporcionados por el INEGI en colaboración con la secretaría de Economía y Asociación Mexicana de Secretarios del Desarrollo Económico (AMSDE) para el año 2018 en México existían un total de 4 millones 169 mil 677 Micro, Pequeñas y Medianas Empresas (MIPyMes), clasificadas en los sectores de manufacturas, comercio y servicios privados no financieros (Líder Empresarial, 2019). Como se puede apreciar la economía del país presenta gran influencia por la existencia de las MiPyMes, quienes generan un gran número de empleos, por lo que existe preocupación por parte de las autoridades para la preservación de estas ya que brindan estabilidad y subsistencia para las familias mexicanas.

A continuación, se muestran y analizan los diferentes apoyos económicos-fiscales implementados por los niveles de gobierno (Federal y Estatal) demostrando el grado de compromiso para con la sociedad mexicana y en particular por las MiPyMes. Se pone en evidencia que han sido insuficientes las medidas implementadas para combatir dicha problemática, lo que deriva en una crisis económica generalizada en todo el país, afectando a este importante sector considerablemente y provocando el cierre de muchas de estas. Después se explica la estrategia metodológica y por último las conclusiones.

\section{SITUACIÓN ECONÓMICA EN MÉXICO ORIGINADA POR LA PANDEMIA COVID-19}

La crisis económica que se está viviendo en el mundo a causa de la pandemia Covid-19 que 
dio inicio en el pasado diciembre 2019 en China, se ha extendido en todos los espacios de la Tierra, propagando sus síntomas a la población, generando problemas respiratorios, fiebre, etc., la situación puede agravarse a tal grado que imposibilita el respirar, provocando la muerte de miles de personas en lo que va del año y al no tener una cura o vacuna aún, el nivel de incertidumbre crece aún más (OMS,2020b). Los datos COVID-19 pueden ser consultados en el Coronavirus Resource Center (JHU, 2020), donde expertos de Johns Hopkins University en salud pública mundial, enfermedades infecciosas y preparación para emergencias han estado a la vanguardia en cifras internacionales. En México en el tablero de datos coronavirus (Gobierno de México, 2020c) desarrollado por CONACYT (Consejo Nacional de Ciencia y Tecnología).

Los estragos de dicha pandemia se logran visualizar en todo el mundo en donde según Forbes (Chacón, 2020) hubo una contracción del 4.8\% en el PIB de Estados Unidos durante el primer trimestre de 2020, siendo el país más poderoso durante muchos años, está afectado por el panorama de resguardo obligatorio de la población; originando que todos los sectores pararan su producción ocasionando un caos económico, nunca visto desde la gran depresión.

El Fondo Monetario Internacional (FMI) proyectó "las contracciones económicas de este año mencionando a USA (-5.8\%), Japón (-5.3\%), Alemania (-6.3\%), Brasil (-5.3\%), México (-6.6\%) y China que pasó de crecer un 6.1\% en 2019 a un $0.5 \%$ en 2020" (Chacón, 2020).

Se visualiza que todas las grandes potencias tendrán una recesión durante este año; y México, como país dentro del grupo de las economías en desarrollo, no es la excepción, ya que se prevé una contracción económica de hasta 6.6\%; porque "en los dos primeros meses de 2020 se generaron 192,094 empleos formales, pero desde el 18 de marzo hasta el 28 de abril se han perdido 707,055" (González, 2020). Los datos son muy desoladores para la economía del país, donde difícilmente se podrá recuperar. El control para disminuir el número de contagios se encuentra relacionado con el cierre de negocios y a su vez con la pérdida de empleos, en donde se puede concluir que entre mayor sea el control de la propagación del virus, mayor son los efectos económicos negativos que se ocasionan; 
afectando algunos sectores más que otros.

Se proyectó el regreso a la llamada "Nueva normalidad", como fue nombrado el programa a seguir para la reapertura de las actividades socioeconómicas del país en tres etapas (Fabila, 2020):

1) Inicio el 18 de mayo para todas aquellas zonas libres de contagio, en donde iniciarán todas sus actividades.

2) Se desarrolló del 18 al 31 de mayo y es a través de protocolos sanitarios, para el reinicio seguro de actividades, consiste en la desinfección e higiene del espacio laboral.

3) A partir del 1 de junio, se clasificará a todo el país con un semáforo con el cual se medirá el nivel de riesgo de la zona tomando en cuenta los contagios que haya.

Esto es un protocolo a seguir que había implementado la Secretaría de Salud, en donde mostraban el actuar del país ante este panorama, pero realmente no se logró aplicar ya que en lo que se pensaba sería la última etapa, en donde se clasificaban a los Estados de acuerdo al nivel de contagios, no fue favorable reactivar la economía. Por las características particulares de los Estados y regiones, los contagios avanzan de diferente manera, por lo que continúan con las acciones de control de la pandemia de mitigación como son cierres y aislamientos masivos, uso de cubrebocas obligatorio, el distanciamiento social y el lavado de manos y superficies provocando que la gran mayoría de las personas permanezca en sus casas limitando la reapertura de negocios y demás actividades.

Ante este panorama fue publicado por las autoridades sanitarias a partir del 30 de marzo de 2020, la suspensión de las actividades consideradas como no esenciales en los sectores públicos, privados y sociales.

A continuación, se enuncian las actividades esenciales en donde no deberían de reunirse más de 50 personas y cumplir todas las medidas preventivas (uso de cubrebocas, caretas, etc.).

1) Las que de manera directa son necesarias para atender la emergencia sanitaria (hospitales, sector farmacéutico, etc.) 
2) Las involucradas en la seguridad pública, defensa de la ciudadanía para mantener el orden.

3) Los sectores considerados como esenciales para el funcionamiento de la economía:

- $\quad$ Financieros

- $\quad$ Recaudación tributaria

- Distribución y venta de energéticos, gasolineras y gas

- Generación y distribución de agua potable

- Industria de alimentos y bebidas no alcohólicas

- Mercados de alimentos, supermercados, tiendas de autoservicio, abarrotes y venta de alimentos preparados

- $\quad$ Servicios de transportes de pasajeros y carga

- $\quad$ Producción agrícola

- $\quad$ Producción pesquera y pecuaria

- $\quad$ Agroindustria

- $\quad$ Producción química y de productos de limpieza

- $\quad$ Ferreterías

- $\quad$ Servicios de mensajería

- Guardias en labores de seguridad privada

- Guarderías y estancias infantiles

- Asilos y estancias para personas de la tercera edad

- Refugios y centros de atención a mujeres víctimas de violencia, sus hijas e hijos

- Telecomunicaciones y medios de información

- $\quad$ Servicios privados de emergencia

- Servicios funerarios y de inhumación

- De almacenamiento y cadena de frío de insumos esenciales

4) Logística (aeropuertos, puertos y ferrocarriles), así como actividades cuya suspensión pueda tener efectos irreversibles para su continuación.

5) La operación de los programas sociales del gobierno. 
6) La conservación y mantenimiento de infraestructura crítica que asegura la producción y distribución de servicios indispensables (agua potable, gasolineras, transporte público, electricidad, etc.) (Gobierno de México, 2020d).

De acuerdo con el boletín emitido por el Senado de México menciona con datos del INEGI, donde "4.1 millones son Pymes del total que representa 95.4 por ciento, de estas 3.6 son pequeñas y 0.8 medianas. Su contribución es de 52 por ciento del PIB. Las pequeñas y medianas empresas generan el 72 por ciento de empleos en México". (Senado de México, 2020, párr. 8).

Son estas de las más afectadas, ya que no cuentan con los recursos necesarios para sostener los gastos de nómina, renta y servicios de sus empresas, puesto que la generación de ingresos está afectada considerablemente.

\section{II.1 Apoyos económicos ante la pandemia}

De acuerdo con datos del Banco Mundial existen hasta el momento 75 países que están brindando apoyos directos a su población por el coronavirus, pero México no está dentro de ellos, para el 20 de marzo el Banco de México (Banxico) sólo anunció "la disminución del depósito de regulación monetaria por un monto de 50,000 millones de pesos para proveer liquidez "y mejorar el funcionamiento de los mercados nacionales" (IDConline, 2020).

En el caso de México existen muchos organismos preocupados por la falta de interés de las autoridades por brindar un mayor apoyo a las MiPyMes y es el caso de la "Confederación Patronal de la República Mexicana (COPARMEX) quienes definieron que México, se encontraba en un escenario de nulo crecimiento (0.1\% para 2019) y de expectativas de crecimiento estimadas en alrededor de $1.2 \%$ a principios de 2020 (Coparmex, 2020a).

De acuerdo con Data COPARMEX, solo el $13 \%$ de empleadas y empleados podrían ser solventes durante una cuarentena de tres meses. Además, cerca del 50\% de las MiPyMes ya se ven afectadas. De no implementarse las medidas diseñadas y sugeridas al Gobierno Federal, para enfrentar la crisis sanitaria y económica, la cual se enfoca en dos grandes prioridades como son la protección del empleo y el ingreso de las familias mexicanas y la recuperación rápida de la economía (COPARMEX, 2020b), "las MiPyMes -que emplean al 
90\% del sector formal del país- estarían en riesgo de subsistir y, con ello, la mayoría de las y los trabajadores verían amenazada su subsistencia”. (COPARMEX, 2020a).

\section{II.2 Apoyos del gobierno federal}

Las medidas implementadas en el país por el gobierno federal son para intentar contrarrestar la crisis económica actual ocasionada por la pandemia y el cese de negocios, para evitar el cierre por completo de las fuentes de empleo y de esta forma evitar consecuencias mayores.

Los tres programas que ha puesto en marcha el gobierno federal para las empresarias y empresarios que no pueden sostenerse ante una pandemia como la que se está atravesando, se enfocan tanto a la economía formal como la informal, se presentan a continuación:

“Crédito Solidario a la Palabra” el cual consiste en otorgar préstamos de $\$ 25,000$ a empresarias y empresarios formales que se encuentren al corriente en sus obligaciones con el IMSS, con esto se refiere al pago puntual de las cuotas patronales, registro de sus trabajadores ante el IMSS y que no hayan despedido durante la contingencia a sus trabajadores. Deberán registrarse y demostrar que cumplen con los requisitos antes mencionados otorgándoles préstamos, empleando las cuentas que ya se tienen registradas en los sistemas.

En dicho programa se tiene 645 mil empresarios que mantienen a sus trabajadores con IMSS. Más de 127 mil aprobados, 90\% entregados que corresponde a más de 2 mil 800 MDP (Gobierno de México, 2020b).

Es un incentivo para los empresarios que llevan al corriente el pago de sus obligaciones y quienes otorgan seguro a sus trabajadores como lo define la ley; brindándoles financiamiento y facilidades de pago para que puedan solventar algunos de los gastos tan fuertes que puede representar el no tener ventas.

"Crédito a la Palabra" consiste en el otorgamiento de préstamos por parte de las autoridades de $\$ 25,000$ para el caso de las empresas informales que se encuentren registradas en el censo de bienestar. El censo de bienestar fue un programa implementado 
por la Coordinación de desarrollo de la oficina de la Presidencia, fue financiado con recursos del fondo de transición después de que entrara al poder el presidente actual (Andrés Manuel López Obrador). Donde 17,860 servidores de la Nación participaron en dicho censo, con 55 millones de cuestionarios. Visitaron un total de 26 millones de domicilios, ubicados en 300,898 localidades y colonias de 2,465 municipios. El censo, refirió, se levantó en un lapso de 6 meses con 20 días (Gobierno de México, 2020a).

Los requisitos para acceder a este crédito es el estar registrado en el censo de bienestar, ser mayor de edad, tener un negocio micro o pequeño con un mínimo de 6 meses de operación, que su actividad se encuentre relacionada con comercialización, producción de bienes o servicios de cualquier tipo de sector con excepción del agropecuario y ubicarse dentro de las zonas que abarca el programa; es decir en las zonas del país donde haya más afectaciones económicas por la pandemia.

Un servidor público se comunicará con los empresarios ya sea de forma presencial o vía telefónica corroborando los datos registrados en el censo, a todos aquellos que reúnan los requisitos ya antes mencionados, les preguntaran si desean acceder al préstamo. En caso de no recibir la notificación las personas interesadas podrán acudir a las oficinas estatales de la Secretaría de Bienestar de su estado, para solicitar información.

Si reúnen los requisitos necesarios los servidores públicos les informarán cuándo y dónde se les deposita dicha cantidad.

El préstamo de "Crédito a la palabra" cuenta con 900 mil aprobados, pequeñas empresas sector comercio, servicios y producción y más de 21 mil entregados por 542.5 MDP (Gobierno de México, 2020b).

El proceso de pago de los préstamos financieros ya antes mencionados se les otorga tres meses de gracia y al cuarto mes comenzarán a realizar los pagos correspondientes los cuales serán de $\$ 850$ durante 36 meses con una tasa de interés de $6.5 \%$ anual.

El nivel de confianza que el gobierno está brindando a los empresarios con el otorgamiento de los préstamos en donde no existe ningún tipo de compromiso que garantice el pago de la obligación adquirida, en donde solo exhortan a la población ante una obligación moral, en 
donde se requiere que realicen los pagos de forma oportuna para posteriormente más préstamos sean otorgados a otros empresarios y el nivel de beneficio se extienda.

Por último, se encuentran los "Microcréditos para el Bienestar" o conocido como "Tandas para el bienestar", lo que ha beneficiado a 600 mil micros negocios locales, 3 mil 400 millones de pesos y más de 200 mil se entregaron en mayo (Gobierno de México, 2020f).

Cabe destacar que este proyecto ya se encontraba en funcionamiento desde que inició su administración en 2019, ya que forma parte de los programas mencionados en el Plan Nacional de Desarrollo 2019-2024, que fueron una serie de medidas que aplicarían a favor de la ciudadanía.

Este programa consiste en otorgar cuatro diferentes tipos de préstamos, la diferencia radica en el monto de cada uno, ver tabla 1.

\section{Tabla 1}

Funcionamiento del programa "Tandas para el Bienestar"

\begin{tabular}{|l|l|c|l|c|}
\hline Apoyo & Monto del & Monto del & Número de & Periodo de gracia \\
apoyo & reembolso mensual & mensualidades & inicial \\
para & reembolso & Tres meses \\
\hline Primero & $\$ 6,000.00$ & $\$ 600.00$ & 10 & Tres meses \\
\hline Segundo & $\$ 10,000.00$ & $\$ 1,000.00$ & 10 & Un mes \\
\hline Tercero & $\$ 15,000.00$ & $\$ 1,500.00$ & 10 & Un mes \\
\hline Cuarto & $\$ 20,000.00$ & $\$ 2,000.00$ & 10 & \\
\hline
\end{tabular}

Fuente: (Gobierno de México, 2020e).

Los periodos de gracia aumentaron por la pandemia, como medida de apoyo por parte de las autoridades, ante la falta de liquidez que existe en el país. El número de apoyos que puede recibir una misma persona es de cuatro (uno inicial y tres subsecuentes), en donde una vez liquidado uno y demostrando que se encuentra al corriente en sus pagos, solicita otro, este se autorizará de inmediato.

Los requisitos necesarios para incorporarse al programa es el llenado de la solicitud, proporcionar información general tanto de la persona como del negocio en donde lo implementará, identificación vigente, CURP y comprobante de domicilio (no mayor a tres meses). 
Pueden participar los microempresarios o incluso los jóvenes que participaron en Jóvenes Construyendo el Futuro (JCF), en donde dicho programa tiene como objetivo enseñarle a un joven "nini”" un oficio para que sea capaz de emprender un negocio.

Al inicio de su mandato el presidente de la República implementó una serie de programas no para empresarias y empresarios entre los que destacan "Jóvenes Construyendo el Futuro" y "Sembrando vida" los cuales consisten en:

Jóvenes construyendo el futuro consiste en "vincular a jóvenes de entre 18 y 29 años, que no estudian y no trabajan, con empresas, talleres, instituciones o negocios donde desarrollan o fortalecen hábitos laborales y competencias técnicas para incrementar sus posibilidades de empleabilidad a futuro. Durante la capacitación, hasta por un año, los jóvenes reciben un apoyo mensual de $\$ 4,310.00$ y un seguro médico contra enfermedades, maternidad y riesgos de trabajo" (Gobierno de México, s.f.).

Sembrando vida consiste en brindar un apoyo mensual de \$5,000; a los campesinos, ejidatarios, comuneros y pequeños propietarios con la finalidad de que estos reforestan y trabajen en el campo, creando sistemas productivos agroforestales el cual combina la producción de los cultivos tradicionales en conjunto con árboles frutícolas y maderables, y el sistema de Milpa Intercalada entre Árboles Frutales (MIAF), con lo que se contribuirá a generar empleos, se incentivará la autosuficiencia alimentaria, se mejorarán los ingresos de las y los pobladores y se recuperará la cobertura forestal de un millón de hectáreas en el país (Gobierno de México, 2019, p.32).

Con respecto a estos programas seguirán actuando de forma habitual durante la contingencia en donde cada beneficiario de estos recibe $\$ 3,748$ y $\$ 5,000$ respectivamente, como medida de apoyo para que la población siga teniendo una forma de subsistir ante la contingencia.

Como iniciativa de la administración actual, referente a las trabajadoras del hogar, el cual consiste en otorgar seguro a este sector ya que se encuentran ante un cierto nivel de riesgo al desempeñar su trabajo y quienes no contaban con ningún apoyo por parte de las autoridades en donde se buscaba responsabilizar a los patrones de la seguridad y bienestar 
de ellas. Ante esta nueva disposición se ha logrado incorporar dentro del programa a las trabajadoras domésticas al programa de créditos solidario a la palabra; ya que por el simple hecho de estar inscritas tienen la posibilidad de recibir el financiamiento de $\$ 25,000$ (Expansión política, 2020b).

De esta forma lo que se busca es seguir apoyando a los miles de hogares que cuentan con este tipo de servicio y a causa de la pandemia no tengan la necesidad de despedir o abstenerse de los servicios a las personas y seguir ofreciéndoles un empleo.

También se busca apoyar a los trabajadores independientes que tengan asegurado a sus trabajadores, podrán acceder al crédito solidario a la palabra siempre y cuando demuestren que se encuentran al corriente de sus obligaciones con las autoridades fiscales y de seguridad social.

Cabe mencionar que el Banco Mundial considera que la implementación de programas como "Jóvenes Construyendo el futuro" son acciones adecuadas para la reactivación de este gran sector, aplicándose en diversos países de Latinoamérica como lo son Colombia con “Jóvenes en acción” y Perú con “Pro jóvenes” (Alistair, 2020).

Una vez expuestos y analizados los diversos apoyos implementados por las autoridades federales se puede observar que resultan insuficientes, ya que los programas no son para combatir la contingencia, solo se enfocan en algunas áreas vulnerables del país, pero no a las MiPyMes.

\section{II.3 Apoyos del gobierno estatal}

En este apartado se presentan los apoyos que otorgaron las autoridades estatales a causa de la pandemia, en beneficio a diferentes sectores.

Tabla 2

Apoyos económicos y fiscales en los diferentes Estados

\begin{tabular}{|l|c|}
\hline $\begin{array}{l}\text { Estados de la república } \\
\text { mexicana } \\
\text { gobernadores }\end{array}$ & \multicolumn{1}{c|}{ Apoyos económicos y fiscales } \\
\hline $\begin{array}{l}\text { Tamaulipas } \\
\text { Francisco García Cabeza }\end{array}$ & $\begin{array}{l}\text { - Subsidio al pago del servicio de agua potable } \\
\text { en los meses de abril y mayo en aquellos hogares }\end{array}$ \\
\hline
\end{tabular}




\begin{tabular}{|c|c|}
\hline $\begin{array}{l}\text { Estados de la república } \\
\text { mexicana } \quad y \\
\text { gobernadores }\end{array}$ & Apoyos económicos y fiscales \\
\hline de Vaca & $\begin{array}{l}\text { que estén al corriente en sus pagos y que su } \\
\text { consumo mensual sea de hasta } 5 \text { metros cúbicos. } \\
\text { - Se pospondrá el pago de los créditos del } \\
\text { programa Inversión Tamaulipas para los } \\
\text { próximos } 90 \text { días naturales, sin recargos e } \\
\text { intereses moratorios. } \\
\text { - Un fondo de } 60 \text { millones de pesos para apoyar } \\
\text { a más de } 20,000 \text { comerciantes semifijos en la } \\
\text { entidad, a fin de que permanezcan resguardados } \\
\text { en sus casas. } \\
\text { - Se diferirá el pago del impuesto del } 3 \% \text { sobre } \\
\text { nómina correspondiente a abril y mayo a todas } \\
\text { las empresas que hasta el primer trimestre del } \\
\text { año tengan registrados menos de } 20 \text { trabajadores. }\end{array}$ \\
\hline $\begin{array}{l}\text { Ciudad de México } \\
\text { Claudia Sheinbaum }\end{array}$ & $\begin{array}{l}\text { - } 50,000 \text { créditos de } 10,000 \text { pesos al que pueden } \\
\text { acceder microempresarios, con un plazo de pago } \\
\text { de } 24 \text { meses, un periodo de gracia de cuatro } \\
\text { meses y a una tasa de interés ordinaria de } 0 \% \text {. }\end{array}$ \\
\hline $\begin{array}{l}\text { Sonora } \\
\text { Claudia Pavlovich }\end{array}$ & $\begin{array}{l}\text { - } 50 \% \text { de descuento en el pago de impuestos } \\
\text { sobre la nómina para empresas con } 50 \text { empleados } \\
\text { o menos. } \\
\text { - } 100 \% \text { de descuento en el pago de impuestos } \\
\text { sobre hospedaje. } \\
\text { - Prórroga para pago por revalidación } \\
\text { vehicular. } \\
\text { - Prórroga para pago de licencias para venta de } \\
\text { alcoholes. } \\
\text { - } 50 \% \text { de descuento en el pago de derechos } \\
\text { registrales por inscripción de vivienda. } \\
\text { - Suspensión de actos de fiscalización. }\end{array}$ \\
\hline $\begin{array}{l}\text { Jalisco } \\
\text { Enrique Alfaro }\end{array}$ & $\begin{array}{l}\text { - Autoempleo para personas de entre } 18 \text { y } 65 \\
\text { años, quienes deberán firmar una "carta } \\
\text { compromiso" con la que se les designarán } \\
\text { labores comunitarias, a cambio de un apoyo } \\
\text { económico de } 5,000 \text { pesos mensuales. } \\
\text { - Programa de conservación de empleos para } \\
\text { microempresas de hasta } 15 \text { trabajadores; esta } \\
\text { iniciativa contempla un financiamiento de } 10,000 \\
\text { hasta } 150,000 \text { pesos por empresa, con una tasa de } \\
\text { interés de } 0 \% \text {. } \\
\text { - Apoyos para productores agrícolas (el monto }\end{array}$ \\
\hline
\end{tabular}




\begin{tabular}{|c|c|}
\hline $\begin{array}{l}\text { Estados de la república } \\
\text { mexicana } \\
\text { gobernadores }\end{array}$ & Apoyos económicos y fiscales \\
\hline & $\begin{array}{l}\text { para los beneficiarios no se ha dado a conocer). } \\
\text { - Fondo Emergente Covid-19 Jalisco te protege, } \\
\text { para personas de } 18 \text { a } 65 \text { años, apoyos de } 5,000 \\
\text { pesos, que tengan ingresos afectados por la } \\
\text { contingencia sanitaria }\end{array}$ \\
\hline $\begin{array}{l}\text { Estado de México } \\
\text { Alfredo del Mazo }\end{array}$ & $\begin{array}{l}\text { - Brindar apoyo económico para amas de casa y } \\
\text { artesanos (sin precisar el monto). } \\
\text { - Distribuir } 300 \text { millones de pesos en apoyo a } \\
\text { los productores del campo en la compra de } \\
\text { insumos, fertilizantes y canastas para el } \\
\text { autoconsumo. } \\
\text { - Destinar } 200 \text { millones de pesos para proyectos } \\
\text { productivos y para microcréditos a tasa } 0 \text { y con } \\
\text { un período de gracia de cuatro meses. } \\
\text { - Las empresas que cuenten con menos de } 50 \\
\text { trabajadores al } 31 \text { de marzo del } 2020 \text { tendrán una } \\
\text { reducción del } 50 \% \text { del Impuesto sobre Nóminas } \\
\text { (ISN) de abril del mismo año, siempre y cuando } \\
\text { no disminuyan su plantilla laboral. Este impuesto } \\
\text { deberá ser pagado a más tardar el } 11 \text { de mayo de } \\
\text { este año. }\end{array}$ \\
\hline $\begin{array}{ll}\text { Nuevo León } & \\
\text { Jaime } & \text { Rodríguez } \\
\text { Calderón } & \end{array}$ & $\begin{array}{l}\text { - A empresas de hasta } 10 \text { empleados tres } \\
\text { meses de condonación del ISN; créditos a } \\
\text { MiPymes hasta por } 1 \text { millón de pesos, con plazos } \\
\text { de hasta tres años y una tasa máxima de } 15.5 \text { por } \\
\text { ciento. Además, hoteles, restaurantes, casinos, } \\
\text { cines, bares y centros sociales estarán exentos de } \\
\text { pagar el ISN de abril a junio. }\end{array}$ \\
\hline $\begin{array}{l}\text { Veracruz } \\
\text { Cuitláhuac } \\
\text { Jiménez }\end{array}$ & $\begin{array}{l}\text { - Otorgando créditos a MiPyMes } \\
\text { dispondrá de } 28 \text { millones de pesos para dar un } \\
\text { apoyo único a choferes del transporte público en } \\
\text { su modalidad de taxi, que se vieron afectados por } \\
\text { la contingencia sanitaria del Covid-19. El apoyo } \\
\text { consiste en } \$ 1,000 \text { por conductor. }\end{array}$ \\
\hline $\begin{array}{l}\text { Tlaxcala } \\
\text { Marco Antonio Mena }\end{array}$ & $\begin{array}{l}\text { Pospondrá tres meses los pagos del ISN, } \\
\text { para ser pagados entre julio y diciembre del } \\
\text { 2020. } \\
\text { - No se cobrará el Impuesto sobre } \\
\text { Hospedaje de abril a diciembre del 2020. }\end{array}$ \\
\hline $\begin{array}{l}\text { Colima } \\
\text { José Ignacio Peralta }\end{array}$ & $\begin{array}{l}\text { - Las empresas que tengan menos de } 10 \\
\text { trabajadores exentaran el pago del ISN por los }\end{array}$ \\
\hline
\end{tabular}




\begin{tabular}{|c|c|}
\hline $\begin{array}{l}\text { Estados de la república } \\
\text { mexicana } \\
\text { gobernadores }\end{array}$ & Apoyos económicos y fiscales \\
\hline & $\begin{array}{l}\text { meses de marzo, abril y mayo de } 2020 \text {. En } \\
\text { cambio, con más de } 10 \text { trabajadores podrán pagar } \\
\text { ISN de marzo, abril y mayo en los meses de } \\
\text { junio a diciembre de } 2020 \text { (se cancela el apoyo si } \\
\text { despiden a sus trabajadores). } \\
\text { - Se condona el impuesto sobre Hospedaje } \\
\text { de abril, mayo y junio } 2020 \text {. }\end{array}$ \\
\hline $\begin{array}{l}\text { Zacatecas } \\
\text { Alejandro Tello Cristerna }\end{array}$ & $\begin{array}{l}\text { - Se condona al } 100 \% \text { del ISN a empresas } \\
\text { con menos de } 20 \text { trabajadores, si tienen } 21 \text { o más } \\
\text { la reducción será del } 50 \% \text { y de } 40 \text { empleados en } \\
\text { adelante será del } 30 \% \text {; siempre y cuando no } \\
\text { despidan a su personal. } \\
\text { - Se condona al } 100 \% \text { el impuesto sobre } \\
\text { Hospedaje. }\end{array}$ \\
\hline $\begin{array}{l}\text { Baja California } \\
\text { Jaime Bonilla Valdez }\end{array}$ & $\begin{array}{l}\text { - Se difiere las declaraciones del ISN de los } \\
\text { meses de marzo, abril y mayo a los meses de } \\
\text { octubre, noviembre y diciembre de } 2020 \text { (en caso } \\
\text { de no pagarlo se les cobrará recargos y } \\
\text { actualizaciones). }\end{array}$ \\
\hline
\end{tabular}

Fuente: Elaboración propia (Expansión política, 2020a) (El Economista, 2020).

Cómo se logra visualizar son muy pocos los Estados que implementaron medidas considerables que minimizaran los efectos de la pandemia en la población más vulnerable. La gran mayoría sólo consideró el pago de dos impuestos como son el referente al hospedaje e Impuesto Sobre Nómina (ISN). Ya que brindaban la posibilidad de diferir su pago siempre y cuando no despidieran a sus empleados, lo que sigue siendo un ingreso para el Estado ya que los impuestos seguirán intactos solo que recibirán el pago en una fecha posterior a la acordada sin tener ninguna afectación.

\section{4 Apoyos fiscales ante la pandemia}

Ante la negativa de otorgar mayores estímulos fiscales por parte de las autoridades, los partidos políticos plantearon iniciativas de ley, pero no han pasado de ser solo una propuesta.

Son muy pocas las acciones referentes a este tema y fue en la Ciudad de México donde:

“para gravámenes locales únicamente se prorrogaron los plazos para el 
cumplimiento de las obligaciones fiscales contenidas en el Código Fiscal de la Ciudad de México, consistentes en la presentación de declaraciones y la realización de los pagos que debían efectuarse durante abril de 2020, pudiendo realizarlas hasta el 30 de abril del mismo año sin pago de accesorios de por medio, así como la ampliación del subsidio de la tenencia con vencimiento al mes de abril en dicho acuerdo, y recientemente mediante otro acuerdo ampliado al 30 de junio."

En el caso de las entidades federativas otorgaron exenciones o diferimientos sobre el impuesto de hospedaje, impuesto sobre erogaciones por remuneraciones al trabajo personal o sobre el impuesto sobre nómina. Al declararse la contingencia se les facilitó a las empresas pagarles a sus trabajadores considerando el salario Mínimo General de \$123.22 (Gobierno de México, 2020e).

En el caso de las declaraciones de Personas Morales no se les permitió prórroga, para las Personas físicas que tenían vencimiento en el mes de abril "se les dio oportunidad de que puedan realizar el pago en caso de impuesto a cargo hasta junio, o en su caso hasta el noviembre para los contribuyentes que optan por pagos en parcialidades" también en este mismo caso las devoluciones de saldos a favor las estuvieron depositando en un periodo de 5 días posteriores a la presentación de la declaración” (Martínez, 2020).

Las medidas fiscales adoptadas por las autoridades no representan un apoyo significativo para los sectores económicos e incluso en este aspecto no se enfocaron como lo requiere el grupo de las MiPyMes, ya que como se mencionó con anterioridad este sector tiene un gran impacto en las finanzas del país.

\section{METODOLOGÍA, TÉCNICAS Y MATERIALES EMPLEADOS}

El presente es un estudio cualitativo, ya que "se enfoca en comprender los fenómenos, explotándolos desde la perspectiva de los participantes en su ambiente natural y en relación con el contexto" (Hernández Sampieri, 2018, p. 367). La investigación cualitativa es interpretativa pues pretende otorgarle sentido a los fenómenos o hechos.

El trabajo se limitó a la descripción y análisis de las estrategias implementadas ante la 
problemática ocasionada por la pandemia, realizando una revisión de la literatura existente. Esta inmersión inicial en el campo implicó sensibilizarse con el entorno en el cual se llevó a cabo el estudio.

\section{CONCLUSIONES}

El país se encuentra en el proceso de resguardo y apenas se están implementado la llamada "nueva normalidad"; la gran preocupación es qué ocurrirá con los miles de personas que se quedaron sin empleo o que no tenían los recursos necesarios para subsistir durante la situación generada por la pandemia, donde ocurrieron despidos sin consideración y es por ello que se exige a las autoridades que brinden apoyos a la población más vulnerable.

Del análisis realizado destacan como medidas implementadas por el gobierno federal, solo los financiamientos, que se otorgan solo si se cumplen con las características requeridas, el detalle es que solo podrían acceder si habían participado en el censo de bienestar donde ya se presenta una limitante para la población.

De igual forma el programa de las tandas de bienestar ya se venía implementando con anterioridad y cabe destacar que no fue una medida nueva a causa de la contingencia.

Analizando los apoyos implementados hasta ahora por el gobierno mexicano se puede concluir que los apoyos de financiamiento otorgados a los microempresarios resultan insuficientes.

En general la postura de las autoridades deja mucho que desear ya que tienen un cierto nivel de desapego para con la población, quienes son los más afectados. El presidente continúa con la realización de sus programas de acción que había proyectado desde inicios de su mandato, en donde sí se generarán miles de empleos, pero los cuales solo serán temporales y no solucionaran el problema de fondo y si los contagios siguen en aumento la situación empeorara.

El apoyo brindado por las autoridades es mínimo ya que son préstamos que requieren de su devolución en un periodo determinado, el monto es bajo y solo permitiría subsistir con los gastos básicos, mínimo unos cuantos meses y si las ventas no se restablecen en su totalidad, 
los microempresarios no podrán cubrir esos pagos.

Trayendo un problema mucho mayor porque existirá menos liquidez y solvencia. Provocando que los niveles de delincuencia aumenten. Es por ello que organismos internacionales consideran que de no ser así numerosos empleos productivos y empresas rentables podrían desaparecer, lo que se traduciría en una recuperación económica más lenta y costosa (Grupo Banco Mundial, 2020).

No todas las medidas implementadas por la actual administración se consideran erróneas e insuficientes, el Banco Mundial, menciona que los programas de capacitación de los jóvenes en el área laboral, representan una forma correcta encaminada a la activación de la economía, se está invirtiendo en este gran sector esperando buenos resultados en un futuro próximo.

Con respecto a las medidas implementadas por las autoridades estatales también resultan mínimas, ya que el diferimiento de algunos impuestos no resuelve en nada la crisis económica que existe, dado que solo retrasan el pago de la obligación, pero de esta forma las autoridades seguirán recibiendo su recaudación de manera normal.

Se requiere que el nivel de compromiso del gobierno sea mayor y se interesen en su población en donde como ya se vio los más vulnerables son los MiPyMes, quienes generan miles de empleos y si apoyan a este sector se estaría apoyando a un sin número de familias.

Además, no se han generado estímulos fiscales significativos que permitieran incentivar a los empresarios, a continuar con su actividad. Con la generación de estas gratificaciones empleadas por las autoridades, lo que se busca es disminuir la carga tributaria de los contribuyentes y a su vez sean más los empresarios interesados en emplear dichos estimulo, al no generarse ninguno a causa de la pandemia se prevé primeramente que les afectaría en la recaudación de los impuestos a las autoridades; en cambio con ellos el beneficio sería mayor a un largo plazo, ya que si las empresas no cierran los empleados mantienen sus trabajos y de esta forma contribuirían con el pago de sus impuestos tanto de la empresa como de los trabajadores, se realizarían el pago de cuotas obrero-patronales, y demás beneficios que se generarían en cadena. 
Ante esta pandemia los microempresarios del sector informal son los más afectados ya que por el resguardo de la población, su demanda disminuyó considerablemente, en cambio los trabajadores formales cuentan con diversos elementos de protección social tales como seguro de desempleo y una cobertura de seguro médico, que les permitirá subsistir con los elementos de primera necesidad.

Las diferentes economías del mundo han adoptado paquetes de medidas para contrarrestar la crisis Covid-19, entre los que se destacan garantías de empleo, subsidios salariales, desgravaciones fiscales, financiamiento para capital de trabajo y alivio del servicio de la deuda y del balance. Con dichos apoyos lo que se busca es conservar el mayor número de empleos y de esta forma alcanzar una estabilidad en la economía del país.

Lo que se propone como medida económica, es la implementación por parte de las autoridades en el país, de una asistencia rápida y amplia para contrarrestar los efectos, a través de generación de estímulos fiscales, la modificación de políticas internas para propiciar que los productores mexicanos puedan vender sus productos, para que no exista competencia con la mercancía extranjera y se consuma lo que el país genera y de esta forma la economía comience a fluir y así la generación del empleo siga su curso. Enfocándose siempre en uno de los sectores más importantes del país que son las pequeñas y medianas empresas, las cuales son un elemento clave de la economía y en donde se podrían enfocar primeramente ya que la situación económica actual lo requiere.

\section{REFERENCIAS}

Alistair D. (2020). Global Productivity: Trends, Drivers, and Policies. Advance Edition. Washington, DC: World Bank. License: Creative Commons Attribution CC BY 3.0 IGO. https://openknowledge.worldbank.org/bitstream/handle/10986/34015/9781464816086.pdf

COPARMEX (8 abril 2020a). El Gobierno Federal debe implementar las propuestas de CEPAL ante la presente crisis de COVID-19. COPARMEX. https://coparmex.org.mx/gobierno-federaldebe-implementar-las-propuestas-de-cepal-ante-la-presente-crisis-de-covid-19/

COPARMEX, (5 abril 2020b). Coparmex pide al presidente preservar empleos y apoyar a las MIPYMES. COPARMEX. https://coparmex.org.mx/coparmex-pide-al-presidente-preservarempleos-y-apoyar-a-las-mipymes/

Chacón, J. L. C. (14 mayo 2020). Recesión 2020: Pandemia Económica, Forbes México. https://www.forbes.com.mx/recesion-2020-pandemia-economica/

El Economista (15 abril 2020). Reaccionan con estímulos fiscales y apoyos 26 entidades ante Covid-19. https://www.eleconomista.com.mx/estados/Reaccionan-con-estimulos-fiscales-y- 
apoyos-26-entidades-ante-Covid-19-20200416-0007.html

Expansión política (3 abril 2020a). Estados enfrentan la pandemia con condonaciones, créditos, programas sociales. https://politica.expansion.mx/estados/2020/04/03/estados-enfrentan-lapandemia-con-condonaciones-creditos-programas-sociales

Expansión política, (20 mayo 2020b). Estos son los apoyos del gobierno federal para la contingencia por coronavirus. https://politica.expansion.mx/presidencia/2020/05/20/estos-sonlos-apoyos-gobierno-amlo-contingencia-sanitaria

Fabila, V. (20 mayo 2020). En qué consiste la "nueva normalidad" laboral y de reactivación de actividades. IDConline. https://idconline.mx/laboral/2020/05/20/en-que-consiste-la-nuevanormalidad-laboral-y-de-reactivacion-de-actividades

Gobierno de México (2019). Programa Sembrando Vida. Gobierno de México. Secretaria de Bienestar, http://www.gob.mx/bienestar/acciones-y-programas/programa-sembrando-vida

Gobierno de México (2020a). Censo del Bienestar presentación. Gobierno de México. Programas integrales. http://www.gob.mx/programasintegrales/galerias/censo-del-bienestar-242798

Gobierno de México (2020b). Créditos a la Palabra. Gobierno de México. Secretaría de bienestar. http://www.gob.mx/bienestar/acciones-y-programas/creditos-a-la-palabra-241368

Gobierno de México (2020c). COVID - 19 México. Gobierno de México. http://datos.covid19.conacyt.mx/index.php

Gobierno de México. (2020d). Medidas de seguridad sanitaria. Se declara como emergencia sanitaria la epidemia generada por covid-19. Gobierno de México. https://coronavirus.gob.mx/medidas-de-seguridad-sanitaria/

Gobierno de México (s.f). Programa Jóvenes Construyendo el Futuro. Gobierno de México. Secretaría del Trabajo y Previsión Social. https://jovenesconstruyendoelfuturo.stps.gob.mx/

Gobierno de México (2020e). Pública Diario Oficial de la Federación salarios mínimos para 2020, Boletín No. 022/2019. Gobierno de México, Comisión Nacional de los Salarios Mínimo. http://www.gob.mx/conasami/prensa/publica-diario-oficial-de-la-federacion-salarios-minimospara-2020

Gobierno de México (2020f). Tandas para el Bienestar. Gobierno de México. Secretaría de Economía. http://www.tandasparaelbienestar.economia.gob.mx/

González, L. M. (2020, 10 mayo). Covid-19: Se perdieron 707,000 empleos entre el 18 de marzo y el 28 de abril. El Economista. https://www.eleconomista.com.mx/opinion/Covid-19-seperdieron-707000-empleos-entre-el-18-de-marzo-y-el-28-de-abril-20200510-0078.html

Grupo Banco Mundial (2020). Protección para las personas y las economías: Políticas integradas en $\begin{array}{llll}\text { respuesta } & \text { a } & \text { la } & \text { COVID-19. }\end{array}$ https://openknowledge.worldbank.org/bitstream/handle/10986/33770/147785SP.pdf?sequence $=19$

Hernández-Sampieri, R. y Mendoza, C.P. (2018). Metodología de la investigación: las rutas cuantitativa, cualitativa y mixta. México. McGrawHill.

JHU. (2020). Coronavirus Resource Center. Johns Hopkins University \& Medicine, https://coronavirus.jhu.edu/map.html

Idconline (2020,30 abril). México, ¿el único país que no apoya el empleo en la pandemia?. IDConline, https://idconline.mx/laboral/2020/04/30/mexico-el-unico-pais-que-no-apoya-elempleo-en-la-pandemia 
Martínez, R. A. (3 julio 2020). Estímulo fiscal por Covid-19. IDConline, https://idconline.mx/fiscal-contable/2020/07/03/estimulo-fiscal-por-covid-19

Líder Empresarial. (2019). Presenta INEGI radiografía de MIPyMES en México. Líder Empresarial, https://www.liderempresarial.com/presenta-inegi-radiografia-de-mipymes-enmexico/

Organización Mundial de la Salud (2020a). Brote de enfermedad por coronavirus (covid-19). Organización Mundial de la Salud, https://www.who.int/es/emergencies/diseases/novelcoronavirus-

2019?gclid=CjwKCAiA4o79BRBvEiwAjteoYCJadzy9F121_ari2A6N2QS5JgctHQvHkj6X7cx qu5X8S1XdYgUhahoCO-YQAvD_BwE

Organización Mundial de la Salud (2020b). ¿Qué es el coronavirus?, Organización Mundial de la Salud, https://www.who.int/es/emergencies/diseases/novel-coronavirus-2019/advice-forpublic/q-a-

coronaviruses?gclid=CjwKCAiA4o79BRBvEiwAjteoYIqr06QMuf_XU9fChwEO9gsDKsSB0 vzThDP-95IEPiTqEN5hZ3_3KhoCZVAQAvD_BwE

Senado de México (2020). Pymes, importante motor para el desarrollo económico nacional: MC. Senado de la República, http://comunicacion.senado.gob.mx/index.php/informacion/boletines/47767-pymesimportante-motor-para-el-desarrollo-economico-nacional-mc.html 